\title{
Intraoperative Bone Registration: An Implementation In Orthopaedic Surgery Using Polaris Vicra System
}

\author{
Barbosa, Roberto M. ${ }^{1}$; Serrador, Luís ${ }^{1}$; Santos, Bruno ${ }^{2}$; Silva, M. V. ${ }^{2}$; De Momi, Elena ${ }^{3}$; Santos, Cristina ${ }^{4}$ \\ ${ }^{1}$ University of Minho, Department of Electronic Engineering, Guimarães, Portugal, \{a68363, a68393\}@alunos.uminho.pt \\ ${ }^{2}$ Orthopaedics department, Hospital de Braga, Braga, Portugal \\ ${ }^{3}$ Bioengineering Department, Politecnico di Milano University, Milan, Italy, elena.demomi@polimi.it \\ ${ }^{4}$ University of Minho, CMEMS/DEI, Guimarães, Portugal, cristina@dei.uminho.pt
}

\begin{abstract}
The introduction of the surgical navigation system in the operating room has brought a great development to the surgeries. Nowadays, the navigated surgeries are present in several fields of medicine. This is a technology that requires an optical tracking system to perform the registration procedure during an intraoperative situation. The preoperative plan consists in performing a $3 \mathrm{D}$ reconstruction of the treated region and planning all procedures during the surgery. The registration is an important procedure once it calculates the transformation between the coordinate system of the preoperative plan on the software and the coordinate system of the surgical room in order to be able the visualization of the position and orientation of the surgical instruments in relation to the $3 \mathrm{D}$ model obtained in the preoperative plan. A workflow in 3DSlicer software to perform the registration procedure is presented in this paper with all required steps to achieve the navigated surgery. Also, it is presented the results obtained from the registration in order to evaluate the procedure.
\end{abstract}

Keywords - optical tracking system; image-guided surgery; 3Dslicer

\section{INTRODUCTION}

The surgical navigation is a term introduced in the medical field in 1992. The first surgical navigation was performed for neurosurgery by resorting to software packages developed by different research groups in previous years, which allow to visualize the human head. In the following years, a lot of improvements in this field were noted and it opened the doors to the computer-assisted surgery (CAS) development [1].

With this development in science and technology some complicated surgical procedures, which are common in traditional surgeries, could be avoided. Also, it has increased considerably the accuracy of surgeries which have become less invasive [2].

The CAS implementation gives to the surgeon a great aid to improve their procedures during the surgery. It allows him to get the information about the performed surgical actions in real-time to ensure that all procedures are accomplishing the preoperative plan. It needs a display device connected with the software to show the virtual scene with the surgical instruments and the anatomical model of the patient. CAS requires the data treatment from the magnetic resonance imaging (MRI) or computed tomography (CT) exams, such as three-dimensional reconstruction or image segmentation, so that it is possible to have a 3D model of the desired anatomical structure to be

* This work is supported by the FCT - Fundação para a Ciência e Tecnologia - with the reference project UID/EEA/04436/2013, by FEDER funds through the COMPETE 2020 - Programa Operacional Competitividade e Internacionalização (POCI) - with the reference project POCI-01-0145FEDER-006941. displayed during the preoperative plan and also during the intraoperative situation [2].

The surgical navigation system (SNS) is one of the main tools in CAS. It provides the location and orientation of the surgical instruments with respect to the patient's coordinate system that is shown in real-time on the display to give that information to the surgeon when he is manipulating the instruments during the surgical operation [3]. It allows the surgeon to reach all the planned regions in the preoperative plan even when there is no a direct view of the specific structure. Also, SNS can be useful to define safety zones giving the information to the surgeon when he is reaching one boundary with the surgical instrument.

Using tracking systems and robotic devices in CAS, it leads to an improvement of the surgical field of view and the accuracy of the procedures [4][5].

One of the most used technologies in the surgical navigation systems is the near-infrared tracking technology due to its reliability and accuracy. Its applicability in operating rooms has suffered an increase with the growth of the assisted surgeries by robotic systems. It has been an important equipment to track the surgical instruments in the surgical room [2]. This kind of equipment is a powerful resource to perform the registration procedure, which involves the acquisition of anatomical landmarks, to be possible the tool navigation in real-time [6].

One of the most popular optical tracking systems working with near-infrared technology is the Polaris system, by Northern Digital Inc. (NDI). It has been widely used in medical procedures once it has shown a good accuracy and reliability [7].

This paper presents a workflow in 3DSlicer software to perform the registration procedure. It was used the Polaris Vicra system as an optical tracking system and the modules from 3DSlicer. All required steps to complete the registration are detailed in order to achieve the navigated surgery.

\section{POLARIS VICRA SYSTEM}

\section{A. General description of the system}

The Polaris Vicra system is an equipment from Northern Digital Inc. (NDI) that can measure the positions in 3D space of their specific markers that are attached to an appropriate tool and its accuracy is $0.25 \mathrm{~mm}$ RMS. This system is composed by a set of tools whose position is given by a specific configuration of sphere markers that can be passive or active 
[7]. Both types of tools can be wireless that allows a greater mobility of them. The passive tools consist of retroreflective passive markers which have a retroreflective coating that reflects infrared (IR) light back to its source. On the other hand, active tools emit IR light by incorporated infrared light emitting diodes (IREDs) markers that are activated when the tool's IR receiver detects IR pulses (code) emitted from the illuminators. The Position Sensor is the main component of the Vicra system and it consists of two illuminators and two sensors. The illuminators are arrays of IREDs responsible to emit IR light for the passive sphere markers or to activate the active markers. The sensor collects IR light reflected from the passive markers or emitted from active markers. By measuring the positions of the markers, the position sensor can calculate the transformations of the tools and then transmits this information to the host computer.

\section{B. NDI 6D Architect}

A tool definition file is created for each tool using the NDI 6D Architect software and it must be loaded from the host computer so that the Vicra System can track the tool.

This file includes the geometry of the tool's markers, the tool's manufacturing data, the information of marker and face normals, groups and faces definitions and the parameters to track the tool. It is possible to create the own tool directed to a specific use. A specific configuration of the markers, with the desired position of them and a specific size and shape of the tool, can be defined. This is an important feature of this system that allows that everyone can create a rigid body or a pointer tool to a specific task that the provided tools by NDI cannot satisfy [8].

\section{REGISTRATION}

The registration is the procedure most important in a navigated surgery. In order to have the tool localization relative to patient's reference, the registration allows to put both scenarios in the same coordinate system and thus it ensures the navigated surgery.

Some interventions require the movement of the treated anatomical region during the surgery's procedures. To provide the real-time tracking of surgical instruments continuously, the navigated surgery is the key. The intraoperative plan is adjusted automatically when the patient's body is moved allowing to choose the best position to perform the intervention's steps. To achieve this, the mathematical relationship between both coordinate spaces, as mentioned above, must be calculated using a tracking system.

The preoperative plan has all the information required during an intraoperative situation. This plan is given by a thorough study of the patient condition and after the registration procedure, intraoperatively, the surgeon has access to what he planned while is performing the intervention.

To achieve the most accurate registration procedure possible, the registration procedure is divided in two different steps: point-to-point registration and surface registration. The first one is the simplest step. It consists in matching two sets of points: one is defined preoperatively in the model from the 3DSlicer software (virtual model) and another one is collected intraoperatively by the surgeon resorting to the pointer tool in the patient's anatomical structure (physical model). Once some anatomical regions are hard to reach with precision by the pointer tool, the surface registration will provide a better outcome from registration when applied after point-to-point registration [4].

In the surface registration, the procedure is different. Basically, this procedure matches both models, the virtual model and the physical model intraoperatively. To realize this, the surgeon collects some landmarks over the anatomical structure surface with the pointer tool and after, these collected points are matched with the virtual model on the 3DSlicer software. In the literature, it was concluded that the iterative closest point algorithm demonstrates a better accuracy in the surface registration outcome [10]. This algorithm consists in paring each collected point from the anatomical structure intraoperatively, with the nearest vertex point of the virtual model. It is an iterative method that aims to estimate the transformation that provides the minimal mean square of the distances between pairs.

The 3DSlicer software is a powerful tool to perform these steps of registration. It has a lot of modules that provide these functions and thus, all required steps to perform this registration method will be detailed in the following sections.

\section{3DSLICER SOFTWARE}

The software used in this project was 3DSlicer, an open source software that has a wide application in the computer aided surgery projects. This software provides a lot of predefined modules and allows the user to develop his own module using specific functions for the desired tasks.

The main tools are provided in the toolbar of this software. All the tasks that can be developed with this software are presented in the toolbar under the heading of 'Modules'. The other part of the window is divided in four parts. On the top, the three-dimensional image, where the different models reconstructed from base images are displayed, along three symmetric windows that have color coded planes (axial, sagittal and coronal, respectively) [11].

The acquired images from CT exam of the patient are saved in DICOM format. These images can be automatically transferred to the 3DSlicer interface using the 'DICOM' module or pressing the 'Load DICOM Data' button on the main window. One library is generated and it is possible to choose between complete studies or only the interesting data. The chosen data will appear on the three lower multiplanar windows.

In order to get the region of interest of the patient scan, it is necessary to process and segment the images. The 'Editor' module is used to apply a threshold to the image and a manual modification is done to remove some parts that are not required. After that, a $3 \mathrm{D}$ model is created based on the processed image using the 'Model Maker' module and also, this model can be processed through the 'Models' module [12].

It is necessary to connect the tracking device with the 3DSlicer software since the goal is to perform a navigated surgery. The connection between the software and the position sensor of Vicra is done using the 'OpenIGTLink' module. This module belongs to an extension that must be installed on 3DSlicer: 'IGT'. The definition file of each tool is uploaded on 
the computer and the 3DSlicer can receive the information about the pose of the tool which is being tracked by the optical system.

The registration procedure is then performed using five different modules. To collect some points from the physical model intraoperatively, it is necessary to use the 'Collect Fiducial' module. This module allows the user to collect the points based on the transformation of the tool provided by the 'OpenIGTLink' module that connects the 3DSlicer with the optical tracking system.

To get the transformation matrix of the point-to-point registration step it is used the 'Fiducial Registration Wizard' module. Thus, the transformation matrix is obtained based on the placed fiducials in the virtual model preoperatively and the same points in the physical model collected intraoperatively.

On the other hand, the 'Fiducials-Model Registration' module is used to achieve the transformation matrix resultant of the surface registration. The user must collect more points of the surface of the physical model and this module gives the transformation matrix that transforms the virtual model in order to match perfectly with the collected fiducials from physical model [11].

The obtained transformations must be applied to the model and it could be done using the 'Transforms' module. To make it in a harden way the 'Models' module is used to update the model's data. If it is not done the model appears to be in the right position on the $3 \mathrm{D}$ view but the data is not updated.

At the end, it is possible to perform a navigated surgery where all the instruments are tracked and displayed on the virtual scene. Figure 1 shows the diagram of all steps that must be done to perform a navigated surgery using 3DSlicer.

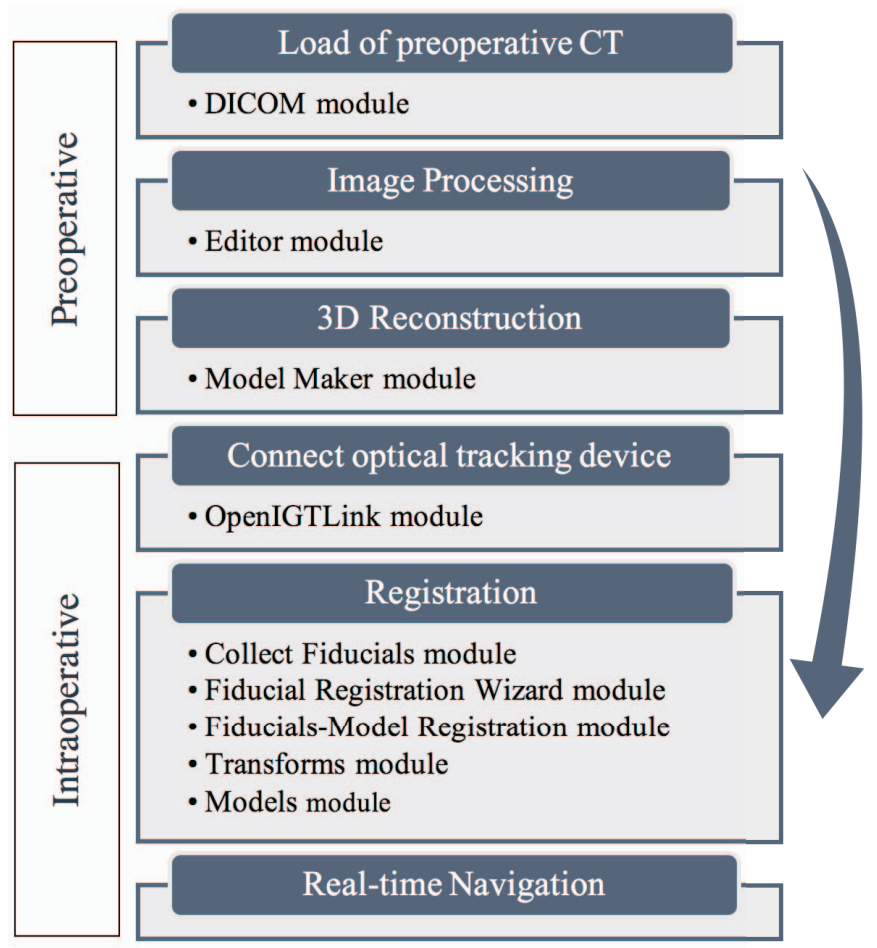

Fig. 1. Workflow to achieve the navigated surgery on 3DSlicer.

\section{MATERIALS AND METHODS}

In this study, it was used a sheep head CT scan. The 3D reconstruction was performed in 3DSlicer following the steps explained on the last section and the physical model used was a $3 \mathrm{D}$ printed skull based on the previously made CT in order to ensure that the dimensions of the physical and the virtual model are the same (fig. 2).

After uploading the definition file of the pointer tool to the host computer, the 'Plus Server Launcher' program is able to read the file and transmit the information about the tool pose to 3DSlicer. Using the 'OpenIGTLink' module, the software is able to display the pointer tool in the $3 \mathrm{D}$ view of the virtual coordinate system. The virtual model is initially placed in an aleatory position and orientation and the pointer tool when placed in the physical model do not reach the virtual model in the 3DSlicer. The goal of registration is to match the virtual and the physical model on the virtual coordinate system so that the tool can reach both models in the same point at the same time.

Now that the tool is connected to 3DSlicer and we have the tool's tip location we are able to perform registration using the modules detailed on the last section. The first thing to do is to collect the same landmarks of the preoperative plan by the same order, i.e., the first fiducial in the preoperative set of points corresponds to the first fiducial in the intraoperative set, and so on. This is done using the 'Collect Fiducials' module where we can press a button whenever we want to record a point of the physical model. Figure 3A shows the landmarks chosen preoperatively in this study.

Now that the preoperative landmarks are defined and the same points were collected from the physical model, it is possible to perform the point-to-point registration using the 'Fiducial Registration Wizard' module. The goal is to match the preoperative landmarks and the points collected intraoperatively. However, the transformation obtained is not sufficient to have a good result of the matching of both models (virtual and physical). The output of this module is the transformation matrix that can transform the landmarks chosen preoperatively into the points collected intraoperatively and to apply it to the model it is necessary to use the 'Transforms' module. As it was mentioned on the last section, this transformation as to be performed in a harden way and to do that, the 'Models' module is used.

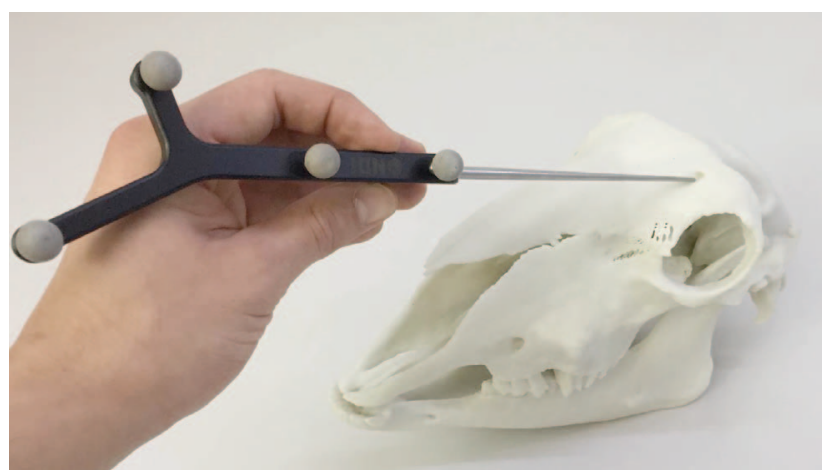

Fig. 2. Passive tool by NDI and the physical model. 

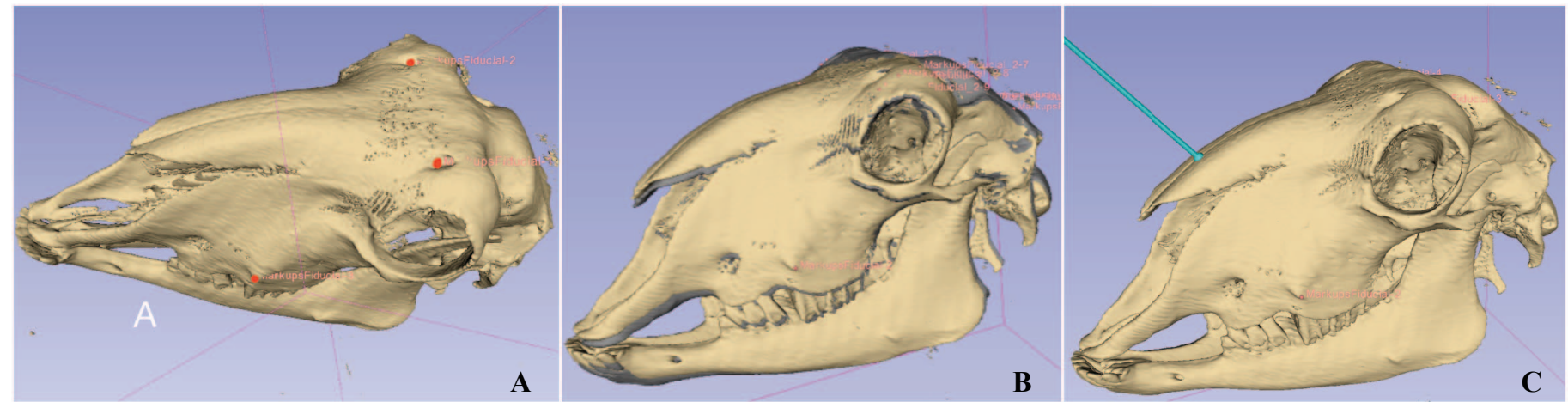

Fig. 3. A) Model in 3DSlicer with chosen landmarks; B) Final result of the registration comparing with the model obtained only by poin-to-point registration; C) Position of the tool in relation to the virtual model (tool navigation).

Since the point-to-point registration is not enough to get a perfect matching of both models, it must be complemented with the surface registration. New points from the physical bone must be collected using the 'Collect Fiducials' module again. To perform the surface registration based on the ICP algorithm the 'Fiducials-Model Registration' module is used. At this moment, the pose of the virtual model is given by the outcome from the point-to-point registration. To match it perfectly with the physical model, it is used together with the new points collected intraoperatively. The output is again a transformation matrix that should match both models perfectly. Like in the point-to-point registration, the 'Transforms' and 'Models' modules are used to transform the virtual model's data and get the final and real pose of that model.

\section{RESULTS}

Figure 3B shows the resultant registration compared to the point-to-point registration. It seems that there is not a big difference between them. However, on a navigated surgery where the surgeon relies on the virtual scene, a simple rotation of the model could guarantee the success of the surgery.

The resultant registration was evaluated based on the distances between the fiducials points collected from physical model to perform the surface registration and the nearest point of the virtual model. As it was possible to ascertain, the average of the distances between the points collected and the nearest point of the model was $0.2719 \mathrm{~mm}$, the standard deviation was $0.1130 \mathrm{~mm}$ and the RMS value was $0.2923 \mathrm{~mm}$. As described above the equipment used to perform the tracking has an accuracy of $0.25 \mathrm{~mm}$ RMS. Analyzing the results, it is possible to verify that the accuracy of the registration procedure obtained is very close to the Polaris Vicra system accuracy which demonstrates a success registration method and its validation. In figure $3 \mathrm{C}$, it is possible to see the final model and the tool navigation.

\section{CONCLUSION}

The registration procedure is a crucial step on navigated surgeries, it allows the surgeon to know where the instruments are, looking for a virtual scene on a display. In surgeries where the field of view is limited due to some muscles or skin of the patient, it can facilitate the surgeon's task and ensure the success of the surgery.

The Polaris Vicra system used as optical system in this study leads to very good results of registration. It is an easy-to- use tracking system that can track a lot of tools and the only requirement is to create the definition of file of each tool.

The 3DSlicer software is an intuitive software that provides all the functions and procedures that are necessary to perform a navigated surgery. It is very intuitive and it allows the user to perform all the preoperative plan, save it and use it during the intraoperative situation. Furthermore, it is an open-source software what means that everyone can develop its own module.

\section{REFERENCES}

[1] J. D'HAESE, J. ACKHURST, D. WISMEIJER, H. DE BRUYN, e A. TAHMASEB, «Current state of the art of computer-guided implant surgery», Periodontol. 2000, vol. 73, n. 25, pp. 121-133, 2017.

[2] K. Cai, R. Yang, Q. Lin, e Z. Wang, «Tracking multiple surgical instruments in a near-infrared optical system», Comput. Assist. Surg., vol. 21, n. 1, pp. 46-55, 2016.

[3] H. Choi, Y. Park, S. Lee, H. Ha, S. Kim, H. S. Cho, e J. Hong, «A portable surgical navigation device to display resection planes for bone tumor surgery», Minim. Invasive Ther. Allied Technol., vol. 0, n. 0, pp. $1-10,2017$.

[4] G. Zheng e L. P. Nolte, «Computer-Assisted Orthopedic Surgery: Current State and Future Perspective.», Front. Surg., vol. 2, n. December, p. 66, 2015.

[5] X. Chen, Y. Lin, C. Wang, G. Shen, S. Zhang, e X. Wang, «A surgical navigation system for oral and maxillofacial surgery and its application in the treatment of old zygomatic fractures», Int. J. Med. Robot., vol. 7, n. April, pp. 42-50, 2011.

[6] C. Tamam e G. G. Poehling, «Robotic-assisted unicompartmental knee arthroplasty.», Sports Med. Arthrosc., vol. 22, n. 4, pp. 219-22, 2014.

[7] R. Decker, A. Shademan, J. Opfermann, S. Leonard, P. Kim, e A. Krieger, «A Biocompatible Near-Infrared 3D Tracking System», IEEE Trans. Biomed. Eng., vol. 9294, n. c, pp. 1-1, 2017.

[8] D. Wallach, G. Toporek, S. Weber, R. Bale, e G. Widmann, «Comparison of freehand-navigated and aiming device-navigated targeting of liver lesions», Int. J. Med. Robot., vol. 10, pp. 35-43, 2014.

[9] Northern Digital Inc., «NDI 6D Architect User Guide», n. March, 2005.

[10] J. R. Cutter, I. B. Styles, A. Leonardis, e H. Dehghani, «Image-based Registration for a Neurosurgical Robot: Comparison Using Iterative Closest Point and Coherent Point Drift Algorithms», Procedia Comput. Sci., vol. 90, n. July, pp. 28-34, 2016.

[11] M. Gonzalo Domínguez, C. Hernández, P. Ruisoto, J. A. Juanes, A. Prats, e T. Hernández, «Morphological and Volumetric Assessment of Cerebral Ventricular System with 3D Slicer Software», J. Med. Syst., vol. 40, n. $6,2016$.

[12] X. Chen, L. Xu, H. Wang, F. Wang, Q. Wang, e R. Kikinis, «Development of a surgical navigation system based on 3D Slicer for intraoperative implant placement surgery», Med. Eng. Phys., vol. 0, pp. $1-9,2017$. 